\title{
ANÁLISIS RETÓRICO-DISCURSIVO DEL GÉNERO ARTÍCULO DE INVESTIGACIÓN EN ESPAÑOL EN EL ÁMBITO DE LA COMUNICACIÓN
}

\section{RHETORICAL DISCOURSE ANALYSIS OF THE RESEARCH ARTICLE GENRE IN SPANISH IN THE COMMUNICATION AREA}

\author{
Bexi Perdomo \\ Universidad de Ciencias y Artes de América Latina (UCAL) \\ bexi.de.flores@gmail.com
}

\begin{abstract}
Resumen:
Objetivo: analizar los rasgos retóricos y discursivos de artículos de investigación de comunicación publicados en español. Metodología: se hizo un análisis de género que integró las premisas metodológicas de la Escuela de la Nueva Retórica Estadounidense, el Modelo Swalesiano y el Modelo Multiperspectiva para análisis de género. Se analizaron artículos publicados por una revista latinoamericana de comunicación indexada en Scopus. Resultados: La estructura predominante es Introducción-Metodología-ResultadosConclusiones (I-M-R-C). Se presentan realizaciones lingüísticas de movimientos y pasos que ilustran las prácticas de escritura. Conclusiones: el análisis de los movimientos y pasos de estas secciones confirmó rasgos propios para el artículo de investigación con diferencias en relación con otras comunidades discursivas.
\end{abstract}

Palabras clave: análisis discursivo, comunicaciones, discurso académico, estructura retórica, artículo de investigación.

\begin{abstract}
:
Objective: to analyze the rhetorical and discursive features of communication research articles published in Spanish. Methodology: it was a genre analysis that integrated the methodological premises of the New Rhetoric School, the Swalesian Model and the Multiperspective Genre Analysis Model. Results: The predominant structure was Introduction-Methodology-Results-Results-Conclusions (I-M-R-C). Some linguistic realizations of moves and steps are included to illustrate writing practices. Conclusions: the analysis of the movements and steps of these sections showed that there are features that differentiate communication research articles from other discursive communities. There is a need for new studies to know other discursive features of this community and its genres. Those studies will be helpful for novel researchers and writers' training.
\end{abstract}

Keywords: discourse analysis, communication, academic discourse, rhetorical structure, research article.

Recibido: 26 de marzo de 2021

Aceptado: 07 de junio de 2021 
Artículo. Bexi Perdomo. “Análisis retórico-discurso del género artículo de investigación en español en el ámbito de la comunicación"

\section{Introducción}

Es importante que los lectores y futuros autores conozcan los rasgos distintivos de los géneros académicos que se publican en su área de conocimiento. En las últimas tres décadas, ha habido un creciente interés por analizar los géneros discursivos, ya que estos permiten conocer las prácticas de escritura disciplinares (Hyland 113). Además, se ha constatado que hay diferencias importantes entre un mismo género en diferentes comunidades disciplinares e idiomas (Saboori y Reza 493). Debido a las implicaciones pedagógicas de estos estudios, los mismos se han constituido en una herramienta de innegable utilidad para la enseñanza de la lectura y la escritura académica (Perdomo 30).

El análisis de género es útil para conocer la manera en que se comunica la ciencia en las disciplinas (Sánchez 20) y para planificar propuestas orientadas a la literacidad académica. Esta literacidad comprende el dominio de la escritura académica disciplinar la cual es una práctica social que permite posicionarse y ocupar roles en la comunidad científica de la disciplina.

Para una exitosa incorporación en las comunidades académicas es preciso conocer y ejercitar sus prácticas (Hyland 124). Para una mejor enseñanza de la producción escrita se necesita saber, además de cómo enseñar y evaluar, qué géneros son pertinentes para los estudiantes a efectos de su incorporación en la comunidad disciplinar. Por lo tanto, para instituciones como la Universidad de Ciencias y Artes de la América Latina (UCAL) (que forma estudiantes en las carreras de comunicaciones y promueve la producción científica en los docentes de la misma área) es preciso conocer estas prácticas y considerarlas en la enseñanza de la escritura académica. Las descripciones de los géneros discursivos (secciones, movimientos y pasos) serán de utilidad para facilitar a los estudiantes y docentes, que se inician como investigadores nóveles, la comprensión de los textos académicos y entender cómo se llevan a cabo los procesos comunicativos en estos géneros (Cotos et al. 104).

Los profesionales de comunicación que incursionan en la investigación científica necesitan fortalecer su escritura académica a partir de estudios discursivos que profundicen sobre las prácticas de esta comunidad y les permitan el desarrollo de competencias de literacidad académica (Navarro 5) que les abre oportunidades para una integración efectiva como la que señala Hyland (126). Diversos autores han reconocido y aprovechado las 
implicaciones pedagógicas del análisis discursivo de género para reforzar la formación universitaria en diferentes carreras (Torres 98; Pérez y Rosado 583). Algunos han logrado incrementar la producción científica en estudiantes y docentes (Morales y Perdomo 270) a través de estas propuestas basadas en análisis de géneros académicos. Sin embargo, no se observan estudios de género sobre las publicaciones científicas en comunicaciones que permitan conocer los hábitos de escritura académica de esta comunidad y ayuden a crear y fortalecer las propuestas de enseñanza como se ha hecho en otros contextos académicos (Torres 95; Pérez y Rosado 581; Morales y Perdomo 270).

$\mathrm{El}$ análisis discursivo de géneros académicos en el ámbito de las comunicaciones es un terreno poco explorado, a diferencia de otras áreas profesionales como la ingeniería (Abarghooeinezhad y Simin 139), contabilidad (Amnuai, The textual 1; Analyses 1), lingüística (Cárcamo 334) y odontología (Morales et al. Estructura 373; Morales et al. Los mecanismos 137), por ejemplo. En la revisión de literatura realizada, los abordajes recientes en comunicaciones en español han sido estudios bibliométricos en España (Compte-Pujol, et al. 1), Colombia (Arroyave-Cabrera 29) y Perú (Estrada-Cuzcano y Alhuay-Quispe 11; Piñeiro-Naval y Morais 113). Estos autores estudiaron la producción académica en el área en el ámbito hispanohablante en revistas indexadas en Scopus. No obstante, sus investigaciones se limitaron a describir la producción científica desde el punto de vista bibliométrico, metodológico y teórico, pero ninguno abordó la caracterización discursiva de géneros particulares.

En cuanto al análisis de géneros discursivos en el ámbito de la Comunicación en español solo se han visto estudios en géneros profesionales, pero no en los académicos y en particular sobre el artículo de investigación (AI, en adelante) también conocido como artículo original y artículo científico. Sal (158) analizó el comentario digital y Campbell y Naidoo (7) determinaron la estructura retórica de 'White papers'. Campbell y Naidoo (21) reconocieron el vacío que existe en cuanto al análisis de géneros en comunicación y la importancia de sus estudios con fines pedagógicos para la producción de diferentes colonias de géneros (profesionales y académicos).

El AI ha sido calificado como uno de los géneros más relevantes en la comunicación científica (Meza 4; Sánchez 22); en consecuencia, conocerlo desde el punto de vista retórico y discursivo es de innegable importancia. Sin embargo, los estudiantes y 
profesionales hispanohablantes que deseen publicar sus investigaciones para la comunidad científica en Comunicación no cuentan con evidencia directamente asociada a este género, dejándolos solos en la tarea de escritura y divulgación. Ante ese vacío, los investigadores nóveles solo cuentan con artículos prescriptivos que no se han basado en estudios textuales y contextuales y que pertenecen a otras disciplinas (Santesteban-Echarri y Nuñez-Morales 3; Mateu et al. 545; Ölçücü et al. 63), lo cual incrementa las posibilidades de fracaso ante la evaluación de pares y, probablemente, frustrando las intenciones de los investigadores nóveles de volver a intentar publicar.

En este contexto, se planteó una investigación cuyo objetivo fue analizar los rasgos retóricos y discursivos de AI en comunicación publicados en español en una revista latinoamericana de alto impacto. Los resultados de este estudio, no solo son útiles para conocer las prácticas discursivas de la comunidad disciplinar de la comunicación, sino que sirven de guía para investigadores nóveles y docentes responsables de la formación en escritura de textos académicos a estudiantes y profesionales de esta comunidad disciplinar, ya que, como señala Skelton (254), este tipo de análisis tiene función orientadora y son de utilidad como insumo para propuestas de enseñanza.

\section{Revisión teórica}

Una comunidad discursiva se define como un grupo de individuos que comparten una formación, ocupación y propósitos comunicativos (por ejemplo, la comunidad de médicos, odontólogos, ingenieros, abogados y profesionales de la comunicación). Según Swales (468) una comunidad discursiva se reconoce como tal si cumple con seis características básicas: (a) comparten objetivos comunes que son de conocimiento público, (b) tienen mecanismos para la intercomunicación entre miembros, (c) usa mecanismos de participación para compartir información relevante al grupo, (d) usan más de un género para llevar a cabo sus objetivos comunicativos, (e) comparten un léxico específico en sus géneros discursivo, y (f) cuentan con miembros reconocidos por su experiencia y experticia en el área (estos marcan tendencias que determinan el destino de los aspirantes y nóveles; por ejemplo, los autores que publican en revistas de alto impacto).

La enseñanza basada en el género parte del estudio de este y favorece el conocimiento de los procesos cognitivos subyacentes en los textos, ofreciendo una visión 
integral de los géneros y prácticas de la comunidad disciplinar en que surgen (Perdomo 31). En la literatura, es cada vez más numerosa la cantidad de artículos en los cuales se muestran las bondades del análisis de género para la enseñanza en el ámbito universitario en diferentes disciplinas en lengua materna y en lengua extranjera (Bhatia, Critical 187; Perdomo y De Jong 175; Perdomo 37; Morales y Perdomo 275; Zare y KeivanlooShahrestanaki 32).

El género discursivo hace referencia a un conjunto relativamente estable de enunciados que comparten contenido temático, lingüístico y estructural que derivan de una comunidad discursiva (Swales 467). Los géneros surgen de las comunidades discursivas cuyos miembros los construyen, interpretan y usan en sus prácticas diarias para el logro de sus propósitos comunicativos en contextos determinados (Bhatia y Salmani 121). La variedad de estos géneros se determina por las posibilidades de la actividad de las comunidades (caracterizadas por su dinamismo y permanente cambio), por lo que su estudio debe ser específico y constante (Miller 60). A partir del análisis de estos géneros es posible la comprensión de las realidades del mundo y las prácticas discursivas de las comunidades en que surgen (Bhatia Critical 188).

Existen colonias de géneros dentro de los que se encuentran los académicos (Bhatia Applied genre 10). Estos representan formas textuales tipificadas propias del contexto de la educación universitaria y la investigación y producción científica en las comunidades discursivas (Berkenkotter 328). Dentro de estas colonias se encuentra el AI, el cual ha sido considerado en la literatura como "un indicador central en cuanto a la producción del conocimiento, el avance científico, el desarrollo disciplinar, y como un elemento que confiere reconocimiento a las instituciones". (Sánchez, 22).

El análisis textual de los géneros discursivos comprende el análisis de su estructura retórica. A partir del estudio de Swales (171) este análisis se hace en tres niveles para cada sección: Movimiento, paso y realización lingüística. Skelton (456) y Arias et al, (151) los explican de la siguiente forma:

- Movimiento retórico: es cada uno de los fragmentos textuales en que se subdividen las secciones del género. El rasgo característico de los movimientos retóricos de una sección es que comparten tanto el contenido semántico como la 
Artículo. Bexi Perdomo. “Análisis retórico-discurso del género artículo de investigación en español en el ámbito de la comunicación”

función comunicativa dentro de esta.

- Paso: es la expresión en que se descomponen los movimientos y se realizan de forma concreta para el cumplimiento de sus objetivos comunicativos.

- Realización lingüística: es un enunciado concreto que se extrae del documento exactamente como el autor lo ha presentado para expresar los pasos.

Skelton (456) señala que no basta saber si un movimiento ocurre o no; es preciso caracterizar la frecuencia y espacio en el que aparece. En este sentido, Skelton (456) presentó criterios fundamentales en el estudio de los movimientos retóricos en un AI para considerarlos obligatorios o característicos:

1. Debe haber un patrón de asociación entre función y su exposición.

2. El rasgo debe haber estado presente en la sección específica del texto (Introducción, Metodología, Resultados, Discusión, por ejemplo) en al menos en $65 \%$ del corpus estudiado.

A partir de esta caracterización es posible acercarse a las prácticas discursivas de la comunidad en estudio y se logra comprender los géneros, según lo que es implícita y explícitamente aceptado por la comunidad académica en que se producen (Skelton 456).

\section{Metodología}

Se planteó un estudio que integró las premisas metodológicas de la Escuela de la Nueva Retórica Estadounidense y el Modelo Swalesiano para análisis de género discursivo y se usó el modelo de análisis multiperspectiva (Bhatia Applied genre 16). Este abordaje comprende el análisis de los textos (análisis textual), y la perspectiva de los miembros de la comunidad para una comprensión integral del mismo (análisis contextual). Este abordaje metodología ha sido seguido en estudios previos en otros contextos académicos (Arias et al. 152; Morales et al., 380).

Corpus: Para la selección de los artículos a analizar se tomó en consideración su impacto en la comunidad científica. En este sentido, una vez consultados docentes 
profesionales de la comunicación, se consideró como fuente la 'Revista de Comunicación'. Esta era la única revista latinoamericana de comunicación indexada en Scopus para la fecha de la realización de la investigación y fue descrita por los informantes expertos como una fuente confiable, de acceso abierto y de consulta frecuente y con prestigio en la comunidad. Es decir, una fuente importante para profesionales y estudiantes de las carreras del ámbito de la comunicación en países latinoamericanos y, por lo tanto, también para aquellos interesados en explorar las prácticas de escritura de esta comunidad disciplinar.

Se analizaron 54 artículos, un corpus adecuado para los objetivos planteados y más amplio que los usados en estudios previos como el de Darabad (125) (con un promedio de 20 por cada una de las tres disciplinas estudiadas), Hanidar (13) ( $\mathrm{n}=40$; de cuatro disciplinas; 10 para cada una), Santiago y Heredero (273) $(n=36)$ y Wahyu (60) $(n=29)$. En palabras, el corpus estuvo compuesto por 481.921. Además, es un corpus suficiente para su propósito, ya que

Mientras que, se recomienda que los corpus para propósitos generales sean lo más grandes posible, las investigaciones que involucran estudio en idiomas técnicos y profesionales han demostrado la representatividad de pequeños corpus de texto de dominio específico, de entre 20.000 y 30.000 palabras..., como consecuencia de las restricciones de los idiomas especializados...: temas predecibles, estructuras sintácticas limitadas de distribución inusual y vocabulario especializado. (Fox 264)

Criterios de selección del corpus: se buscaba analizar el género AI en español, ya que los informantes expertos reconocieron su importancia en la comunidad. Se incluyeron los artículos en español considerados por Scopus para el cálculo del índice de impacto de la Revista de Comunicación en el último período previo al estudio. Estos artículos debían estar en la revista dentro de la sección de 'Artículos'.

Criterios para el análisis: se consideró un análisis completo que comprendió la descripción textual (estudio de la estructura retórica del artículo, identificación de movimientos y pasos retóricos e identificación de realizaciones lingüísticas prototípicas) y la descripción contextual (comprensión del análisis textual a partir de lo expresados por expertos).

Criterios de selección de los expertos: Participaron 10 expertos como informantes de la comunidad. Para llegar a ellos, se revisó el corpus y se identificaron autores que 
además de tener la formación profesional en comunicaciones y haber publicado uno o más artículos que conforman el corpus, mostraran una amplia trayectoria publicando en el ámbito de las comunicaciones en otras revistas y pertenecieran a diferentes países. La participación fue voluntaria, una vez que recibieron la invitación y se les explicó la naturaleza de la investigación. Se plantearon dos formas, sincrónica (video reuniones) y asincrónica (correo electrónico), para el intercambio de información.

Procedimientos: el estudio se llevó a cabo en diferentes etapas. Se hizo la selección del corpus, su codificación y la conversión a formato .txt. Luego, se creó la base de datos para el análisis cuantitativo de los textos y se procedió también al análisis cualitativo y. Finalmente, se realizaron las consultas y entrevistas a los expertos.

Análisis: para el análisis textual se hizo una revisión manual inicial seguida del procesamiento con el software Wordsmith $7.0{ }^{\circledR}$. El análisis cualitativo permitió conocer los rasgos discursivos y comprender las dimensiones sociales, cognitiva y textual de los artículos en el área de la comunicación. El cuantitativo permitió conocer la frecuencia de los movimientos y pasos a fin de poder completar la caracterización según lo planteado por Skelton (456). Los análisis cuantitativos se hicieron usando Excel ${ }^{\circledR}$ e IBM SPSS ${ }^{\circledR}$ V.27.

Para una mejor comprensión de lo observado, se hizo el análisis contextual, con el apoyo de los expertos. Estos fueron consultados sobre los rasgos observados en el corpus y que parecían ser diferenciadores para esta comunidad disciplinar. Sus opiniones fueron comparadas y se observaron las tendencias (convergentes y divergentes) en las respuestas.

\section{Resultados}

Se analizaron 54 artículos, equivalente a 1.129 páginas y 481.921 palabras. La extensión de los artículos en palabras estuvo en un rango de 5.305 - 13.810, con un promedio de 8.924.

Los autores de los artículos procedían en su mayoría de España (65\%) y países latinoamericanos como Perú, Chile, Argentina y México (22\%). Algunos AI fueron publicados por colaboraciones entre autores españoles y latinoamericanos (11\%) y entre autores de países latinoamericanos diferentes $(2 \%)$.

Se observó que $29,6 \%$ de los AI eran de autoría única. Los AI en coautoría presentaron mayores porcentajes para tres y dos autores $(33,3 \%$ y $31,5 \%$, respectivamente). 
Solo hubo dos artículos (3,7\%) con cuatro autores y uno (1,9\%) con cinco. El promedio de autores por artículo fue dos $(\mathrm{SD}=.96)$.

\subsection{Estructura retórica de los artículos}

La estructura retórica de los artículos fue variada (ver Figura 1), aunque la revista propone una (introducción, marco referencial, metodología, análisis de los resultados y, discusión y conclusiones) en las normas para autores. Este hallazgo refleja que, si bien existe una estructura propuesta por la revista como guía, existe flexibilidad para publicar artículos con otras diferentes ajustadas a la naturaleza de los estudios. Así lo confirmaron la mayoría de los expertos entrevistados. Este hallazgo puede explicarse en el planteamiento de Kafes (135) quien señala que mientras más experimentado es el autor, más se arriesga y se sale de los estándares cuando considera necesario. No obstante, dos de estos expertos consideran que este es un aspecto que la comunidad debería modificar y asumir, como otras, una estructura canónica predominante.

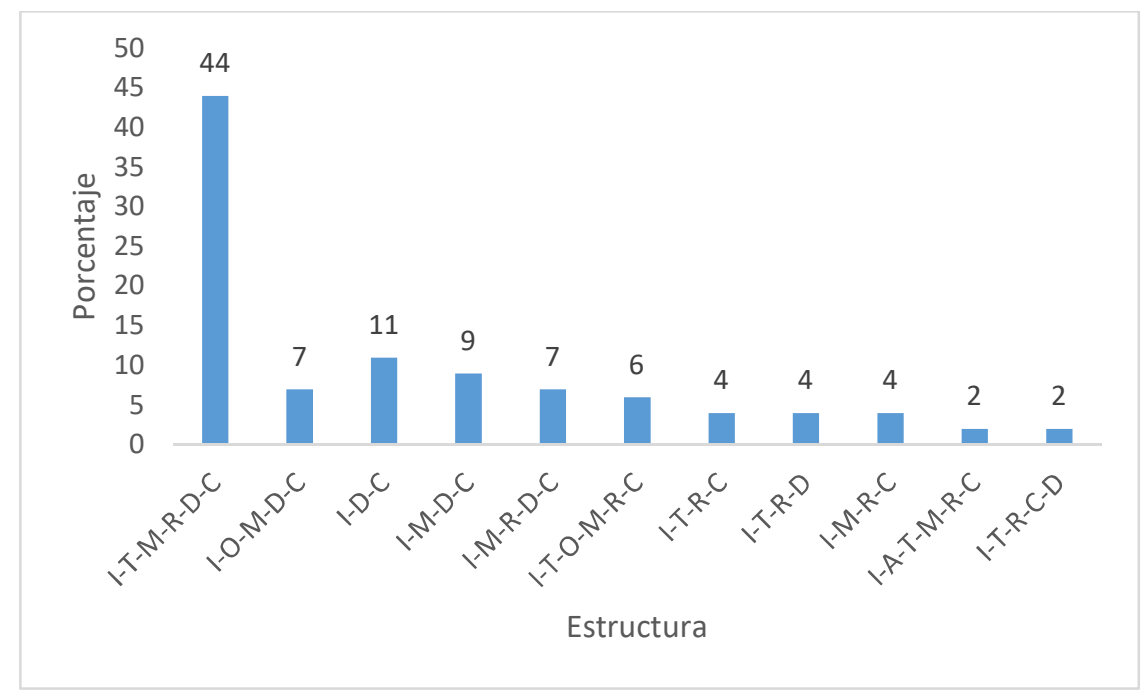

Figura 1. Estructuras retóricas observadas ( $\mathrm{I}=$ Introducción, $\mathrm{O}=$ Objetivo, $\mathrm{T}=$ Teoría, $\mathrm{M}=$ Metodología, $\mathrm{R}=$ Resultados, $\mathrm{D}=$ Discusión, $\mathrm{C}=$ Conclusiones).

Los artículos analizados estaban ubicados en las secciones de 'artículos'. Estos presentaban resultados de estudios de diferentes enfoques de investigación (cualitativa $53,7 \%$, cuantitativa $18,5 \%$ y mixtos $27,8 \%$ ). Dentro de los estudios cualitativos se observaron revisiones tradicionales de literatura con la estructura (Introducción- 
Artículo. Bexi Perdomo. “Análisis retórico-discurso del género artículo de investigación en español en el ámbito de la comunicación"

Desarrollo- Conclusión/I-D-C); en estos, el desarrollo generalmente se compone de categorías debidamente subtituladas sobre las cuales posteriormente se concluye. A diferencia de otras comunidades que tiene una sección para revisiones tradicionales de literatura, estos artículos se presentaban junto a los AI que respondían a otros enfoques y diseños.

Se registró el porcentaje de aparición de las secciones retóricas del AI (Tabla 1) para observar las tendencias a la obligatoriedad, según los criterios de Skelton (456).

Tabla 1. Secciones retóricas de los AI en el corpus analizado (en negrillas los que superan el 65\% de presencia en el corpus).

\begin{tabular}{lr}
\hline Sección & \multicolumn{1}{c}{$\%$} \\
\hline Introducción & $\mathbf{1 0 0}$ \\
Revisión Teórica & 63 \\
Metodología & $\mathbf{8 7}$ \\
Resultados & $\mathbf{8 5 . 2}$ \\
Discusión & 18.5 \\
Conclusiones & $\mathbf{6 6 . 7}$ \\
Resultado y Discusión & 5.6 \\
Discusión y Conclusiones & 25.9 \\
\hline
\end{tabular}

Todos los AI presentaron introducción y se considera obligatoria al igual que metodología (87\%), resultados (85.2\%), siguiendo los criterios de Skelton (456). La sección conclusiones estuvo presente (como sección separada o unida a la discusión) en 94\% de los AI; no obstante, la tendencia más marcada (66.7\%) es presentarlas como sección obligatoria separada. La revisión teórica se considera opcional, aunque muestra tendencia a la obligatoriedad al estar presente en casi el 65\% del corpus.

Los autores usaron diferentes nombres para estas secciones retóricas (Cuadro 1). La sección con más variaciones en su denominación fue la de metodología. Esto pudiera obedecer la diversidad de diseños y tipos de investigación. Algunos informantes expertos señalaron que ellos nombraban esta sección en función de la metodología con la que estaban trabajando. Además, señalaron que no recibieron objeciones al respecto, lo que denota cierta flexibilidad a la formalidad y predominio del contenido más allá de su denominación y que los pares evaluadores se concentran en el fondo, más que en la forma, respetando los criterios de los autores. Algunos informantes señalaban que más allá de la 
uniformidad del término que se use para denotar la metodología, lo importante es que en esta sección se provee la descripción metodológica y que esta sea exhaustiva.

\begin{tabular}{|c|c|c|c|c|}
\hline Introducción & Revisión teórica* & Metodología & Resultados & Conclusiones \\
\hline Introducción & Antecedentes & Método & Resultados & Conclusiones \\
\hline $\begin{array}{l}\text { Supuesto de } \\
\text { partida, } \\
\text { Objetivo y } \\
\text { pregunta de } \\
\text { investigación }\end{array}$ & $\begin{array}{l}\text { Marco referencial } \\
\text { Desarrollo } \\
\text { Estado de la } \\
\text { cuestión } \\
\text { Contextualización } \\
\text { Presupuestos } \\
\text { teóricos } \\
\text { Marco conceptual } \\
\text { Marco teórico }\end{array}$ & $\begin{array}{l}\text { Método y } \\
\text { técnicas de } \\
\text { investigación } \\
\text { Metodología y } \\
\text { selección de } \\
\text { muestra } \\
\text { Metodología y } \\
\text { procedimientos } \\
\text { Objetivo y } \\
\text { metodología } \\
\text { Metodología y } \\
\text { justificación } \\
\text { Estudio empírico } \\
\text { Planteamiento y } \\
\text { metodología } \\
\text { Metodología y } \\
\text { fases de la } \\
\text { investigación } \\
\text { Perspectivas de } \\
\text { análisis } \\
\text { Estrategia de } \\
\text { revisión } \\
\text { Diseño y muestra } \\
\text { Estudio de caso }\end{array}$ & 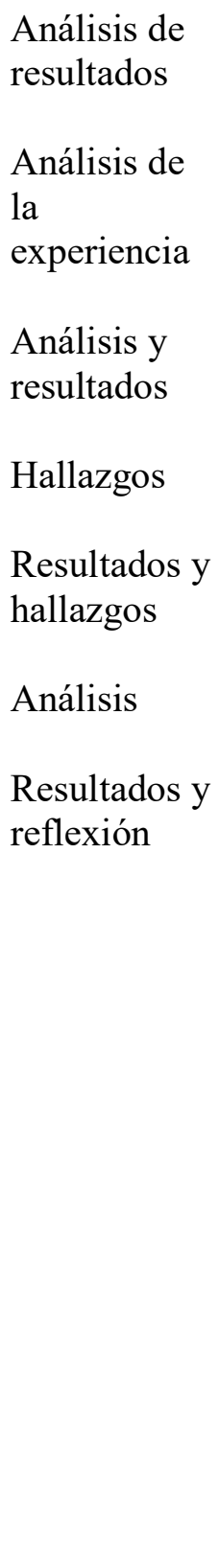 & 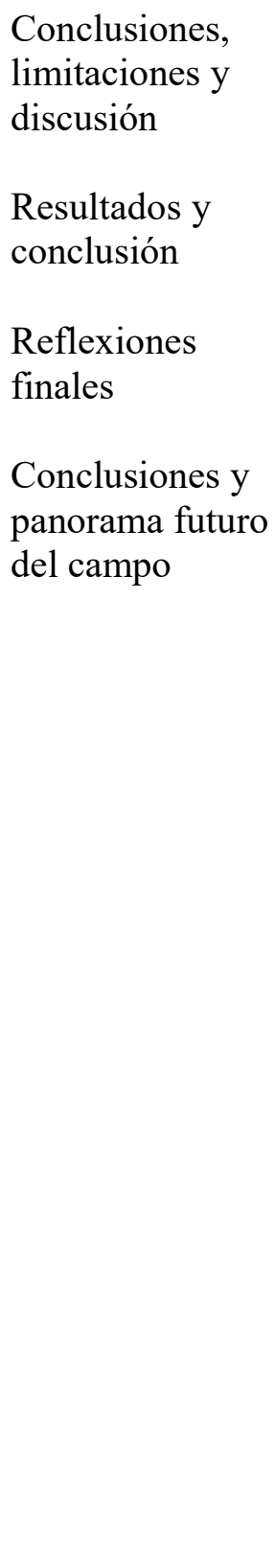 \\
\hline
\end{tabular}

Cuadro 1. Diversas denominaciones dadas a las secciones retóricas predominantes en el corpus. *Se incluye porque es una tendencia que casi alcanza el $65 \%$ en el corpus. 
No fue habitual la presencia de secciones complementarias. Los autores prefieren incorporar figuras (fotografías, y otras expresiones gráficas) dentro del texto para ampliar o apoyar sus ideas y no se apoyan en anexos. También fue poca la presencia de las secciones de agradecimientos y financiamiento, en las cuales los autores suelen hacer mención a la colaboración de instituciones o entes que financian las investigaciones (López et al. 242).

La Tabla 2 muestra todos los movimientos y pasos presentes en cada sección retórica obligatoria. Algunos de los movimientos considerados opcionales presentaron una frecuencia que se aproxima al 65\% que señala Skelton (456).

Tabla 2. Movimientos y pasos en las secciones de los AI analizados.

\begin{tabular}{|c|c|}
\hline Secciones, movimientos y pasos & $\begin{array}{l}\text { Carácter según } \\
\text { frecuencia }\end{array}$ \\
\hline INTRODUCCIÓN & $\begin{array}{l}\text { Obligatorio (100 } \\
\%)\end{array}$ \\
\hline M1 Establecer territorio & $\begin{array}{l}\text { Obligatorio } \\
(94.4 \%)\end{array}$ \\
\hline P1 Hacer generalizaciones del & $\begin{array}{l}\text { Obligatorio (85.2 } \\
\%)\end{array}$ \\
\hline P2 Revisión de literatura previa & Opcional (63\%) \\
\hline M2 Establecer el vacío & Obligatorio (83\%) \\
\hline P1A Contraargumentación & Opcional $(25.9 \%)$ \\
\hline P1B Indicar una brecha & Opcional $(27.8 \%)$ \\
\hline P1C Elaborar una pregunta & Opcional $(24.1 \%)$ \\
\hline P1D Continuar tradición & Opcional $(24.1 \%)$ \\
\hline M3 Llenar el vacío & $\begin{array}{l}\text { Obligatorio } \\
(79.6 \%)\end{array}$ \\
\hline P1A Presentar el objetivo & $\begin{array}{l}\text { Obligatorio (74.1 } \\
\%)\end{array}$ \\
\hline P1B Presentar la investigación & Opcional (37 \%) \\
\hline P2 Presentar principales hallazgos & Opcional $(11.1 \%)$ \\
\hline P3 Indicar la estructura del artículo & Opcional $(9.3 \%)$ \\
\hline REVISIÓN TEÓRICA & Opcional (63\%) \\
\hline M4 Teoría subtitulada & Opcional $(54 \%)$ \\
\hline METODOLOGÍA & Obligatorio (87 \%) \\
\hline M5 Describir el studio & $\begin{array}{l}\text { Obligatorio (85.2 } \\
\%)\end{array}$ \\
\hline P1 Presentar el objetivo & Opcional $(55.5 \%)$ \\
\hline P2 Describir metodología del & $\begin{array}{l}\text { Obligatorio (72.2 } \\
\%)\end{array}$ \\
\hline $\begin{array}{l}\text { M6 Procedimiento de recolección de datos } \\
\text { P1 Describir la muestra }\end{array}$ & $\begin{array}{l}\text { Obligatorio }(87 \%) \\
\text { Obligatorio }(83.3\end{array}$ \\
\hline
\end{tabular}




\begin{tabular}{|c|c|}
\hline & $\%)$ \\
\hline P2 Explicar proced. de recolección & $\begin{array}{l}\text { Obligatorio (83.3 } \\
\%)\end{array}$ \\
\hline P3 Justificar los procedimientos & Opcional $(55.6 \%)$ \\
\hline M7 Describir análisis & $\begin{array}{l}\text { Obligatorio (79.6 } \\
\%)\end{array}$ \\
\hline P1 Describir y justificar análisis & $\begin{array}{l}\text { Obligatorio (68.9 } \\
\%)\end{array}$ \\
\hline LSULTADOS & $\begin{array}{l}\text { Obligatorio ( } 85.2 \\
\%)\end{array}$ \\
\hline M8 Hacer referencia al objetivo & Opcional $(9.3 \%)$ \\
\hline M9 Reportar un resultado & $\begin{array}{l}\text { Obligatorio (94.4 } \\
\%)\end{array}$ \\
\hline M10 Presentar Tablas y Gráficos & $\begin{array}{l}\text { Obligatorio (77.8 } \\
\%)\end{array}$ \\
\hline ONCLUSIONES & $\begin{array}{l}\text { Obligatorio (66.7 } \\
\% \text { ) }\end{array}$ \\
\hline M11 Información previa del estudio & Opcional $(53.7 \%)$ \\
\hline M12 Alusión a resultados generales & Opcional $(63 \%)$ \\
\hline M13 Presentar conclusiones por objetivos & $\begin{array}{l}\text { Obligatorio (83.3 } \\
\%)\end{array}$ \\
\hline M14 Discutir contrastando con otros autores & Opcional $(25.9 \%)$ \\
\hline M15 Exponer implicaciones de los hallazgos & Opcional $(59.3 \%)$ \\
\hline M16 Proponer investigaciones futuras & Opcional $(27.8 \%)$ \\
\hline
\end{tabular}

Nota: La revisión teórica no se incluyó debido a que no alcanzó el 65\% señalado por Skelton (456); sin embargo, se reconoce la tendencia hacia su obligatoriedad.

A continuación, se presentan detalles de los movimientos, pasos y realizaciones lingüísticas de cada una de estas secciones retóricas. Para tal fin de mostrarán ejemplos prototípicos extraídos textualmente del corpus.

\subsection{Introducción}

La sección de Introducción estuvo presente en el 100\% de los artículos. Se observaron los tres movimientos encontrados en el modelo Swales (172) conocido como CARS de (siglas para su nombre en inglés 'create a space for research'): establecer el territorio (94.4\%), establecer el vacío o brecha (83.3\%) y llenar el vacío (73\%). Los detalles de la frecuencia de los pasos para cada movimiento se observan en la Tabla 2.

En el primer movimiento, el paso presente en la mayoría de los artículos fue hacer generalizaciones sobre el tema $(85,2 \%)$. El paso de referir a estudios previos $(63 \%)$ casi 
Artículo. Bexi Perdomo. “Análisis retórico-discurso del género artículo de investigación en español en el ámbito de la comunicación"

alcanza el mínimo señalado por Skelton (456) para ser considerado obligatorio o característico.

En el movimiento 2, establecer el vacío, los autores usaron de forma combinada los diferentes pasos al igual que se ha visto en estudios previos en otras disciplinas (Soodmand et al. 171; Santiago y Heredero 278). La importancia de este movimiento radica en que el mismo implica la justificación de la investigación a través de los diferentes pasos, los cuales se usan incluso combinados para complementarse. El ejemplo (1) muestra el paso elaborar una pregunta y el ejemplo (2) continuar una tradición.

(1) Se inicia con un primer cuestionamiento: ¿qué relación existe entre la percepción que tienen los jóvenes acerca de los estereotipos de género considerando diferentes contextos, en primer término: la sociedad, la publicidad en general y la publicidad de las universidades de la región de Valparaíso; y como segundo aspecto, la percepción individual que tienen los jóvenes estudiantes al respecto. (RDC-V171051)

(2) También, es importante precisar que el presente estudio es consecuente con lo señalado por Luken y Stares (2005), Araya (2006) y Jamali et al. (2009) acerca de la importancia del desarrollo de la investigación de desempeño de la RSE en países en vías de desarrollo. (RCD-V171046)

El movimiento de llenar el vacío se ejecutó básicamente por medio de la presentación del objetivo, independientemente de si este se presentaba nuevamente en la sección de metodología. Los ejemplos (3) y (4) representan la forma en que los autores ejecutaban este paso. Algunos autores presentaban más de un objetivo precedido de una frase introductoria como en el ejemplo (4).

(3) Como objetivo general, nos proponemos comprender la forma en que la academia peruana ha estudiado el fenómeno del fútbol, haciendo hincapié en la arista comunicacional. (RCD-V171045)

(4) Nuestra investigación persigue los siguientes objetivos:

- Proponer un modelo de análisis válido para las herramientas cibernéticas diocesanas, que tenga en cuenta las relaciones entre el entorno corporativo (website) y el entorno público (medios sociales).

- Establecer las dimensiones de la relación entre Iglesia e Internet, observando las diferencias de la Iglesia respecto a otros actores en el panorama comunicacional.

- Estudiar la composición tareas y rutinas de trabajo de las delegaciones .... (RDC-V172031) 


\subsection{Revisión teórica}

La revisión teórica estuvo presente en $63 \%$ del corpus de análisis y si bien no alcanza el porcentaje para considerar permanente u obligatorio, se acerca lo suficiente a este para considerar la tendencia hacia su obligatoriedad. Ningún AI presentó párrafo introductorio en esta sección, sino que iniciaban directo con su presentación y desarrollo el cual, en la mayoría de los casos, se hacía a partir de categorías subtituladas (54\%). Algunos artículos (13\%) presentaban la revisión teórica como parte de la introducción.

\subsection{Metodología}

La sección de Metodología estuvo presente en $87 \%$ de los artículos. La Tabla 3 contiene los porcentajes de aparición de uno de los movimientos y sus correspondientes pasos.

Tabla 3. Movimientos y pasos en la sección de metodología.

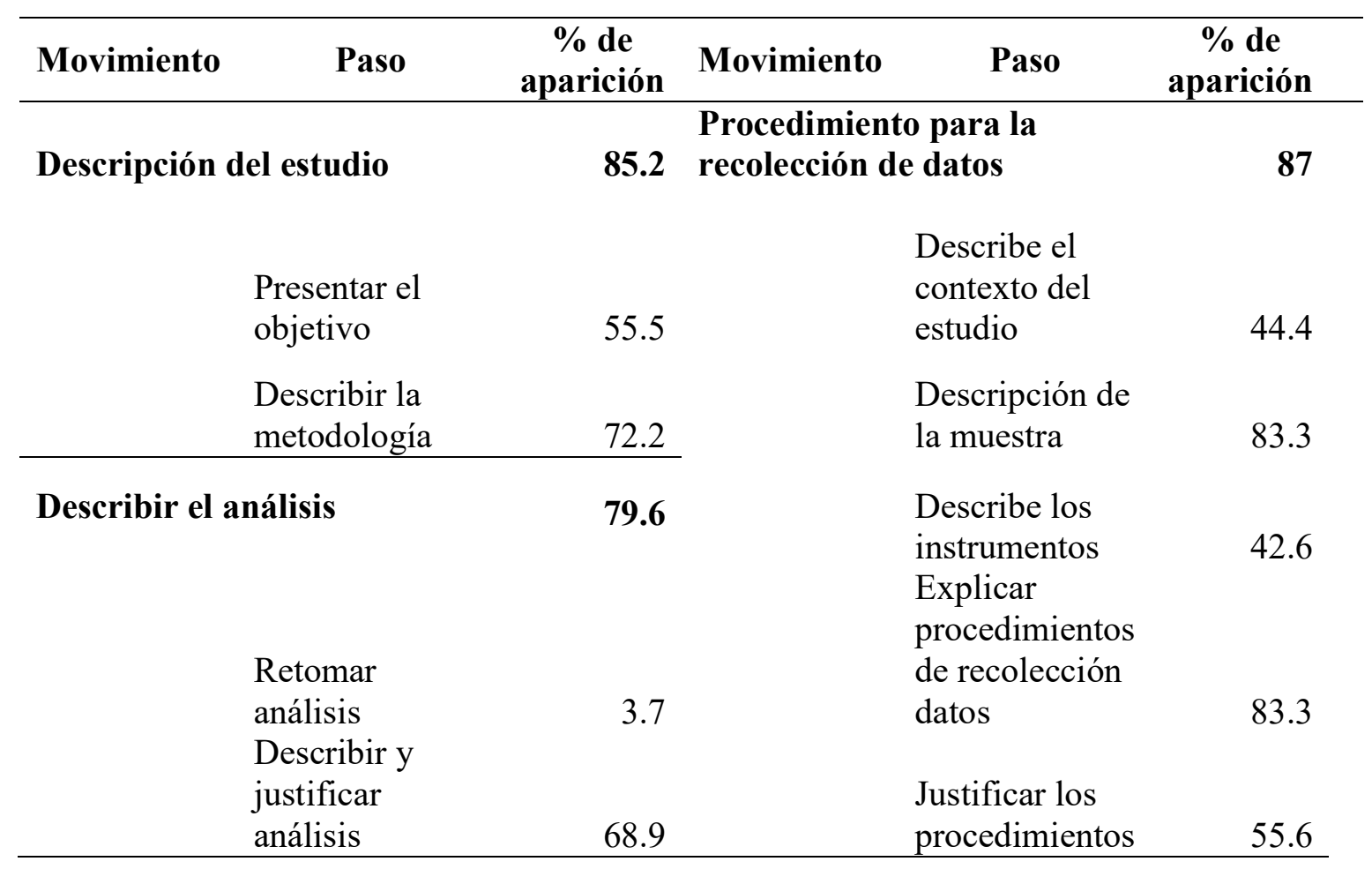


Artículo. Bexi Perdomo. “Análisis retórico-discurso del género artículo de investigación en español en el ámbito de la comunicación”

Se observaron tres movimientos retóricos. El interés por los autores en cuanto a la metodología se orienta a describir: el estudio (85.2\%), los procedimientos para la recolección de los datos (87\%) y la forma en que estos se han analizado $(79.6 \%)$.

Más de la mitad de los artículos (55,5\%) comienza esta sección con la presentación del objetivo (ejemplo 5), independientemente si se trata de un estudio cualitativo o cuantitativo. La mayoría de expertos consideraron oportuno presentarlo al inicio de esta sección porque el mismo (al igual que las hipótesis o supuestos) guían el estudio y es útil como inicio de su descripción. En cuanto a la presentación del diseño, lo hacen aludiendo, de forma muy sucinta, al enfoque, alcance y método (ejemplos 6,7 y 8 ) y se observa que se hace la descripción con y $\sin$ apoyo en la literatura especializada (ejemplos 8 y 9 , respectivamente).

(5) El objetivo principal de esta investigación es identificar los encuadres hegemónicos en el discurso de los partidos políticos y en la cobertura de los medios de comunicación a lo largo de la campaña electoral catalana de 2017 (del 5 al 19 de diciembre). (RCD-V182010)

(6) La presente investigación tiene un carácter exploratorio, cuyos datos y fuentes son abordados a partir de una revisión en términos cualitativos. (RDCV182001)

(7) La presente investigación es del tipo cuantitativo, con alcance de tipo explicativo y correlacional. (RDC-V171046)

(8) Esta investigación corresponde a un tipo de estudio exploratorio-descriptivo de acuerdo a la clasificación de Danhke (Salinas y Cárdenas, 2008) que utiliza como método un análisis de contenido diseñado específicamente para sitios webs (Dader, 2004, 2006, 2008, 2011) basado en una escala valorativa. (RDCV182008)

(9) En cuanto a la metodología utilizada, se plantea un estudio de caso a través de recursos variados - entrevistas, observación participante, cuestionarios o documentos bibliográficos - que permiten una visión holística del objeto de estudio. (RDC-V181028)

En el movimiento descripción de recolección de datos, los pasos con mayor frecuencia fueron descripción de la muestra $(83.3 \%)$ y procedimientos para recolección de datos (83.3\%), seguidos de la justificación de los procedimientos seleccionados (55.6\%). Los siguientes ejemplos ilustran la realización lingüística de la descripción de las muestras y procedimientos para su selección (ejemplos 10 y 11, respectivamente), procedimientos para la recolección de datos (ejemplo 12) y justificación de los procedimientos planteados (ejemplo 13) que si bien no alcanzó en 65\% muestra tendencia a la obligatoriedad. 
(10) La investigación compara los encuadres presentados por seis periódicos (El País, El Mundo, La Vanguardia, El Periódico de Catalunya, El Punt Avui y Ara) en sus titulares con los adoptados por los siete partidos políticos representados en el Parlament de Catalunya (JuntsXCat, ERC, PSC, PP, Ciudadanos, Catalunya en Comú-Podem y CUP) a través de las noticias publicadas en sus páginas web durante la campaña electoral catalana. (RDCV182010)

(11) Para la selección de la muestra se envió el cuestionario a todos los suscriptores de la revista que conforman un universo de 222.000 sujetos, por tanto, se pretendía que toda la población objeto de estudio formara parte de la investigación. (RDC-V181028)

(12) Se desarrollaron entrevistas en profundidad y focus group, diseñando para ello, un guion de tópicos que cubrió con amplitud los objetivos. (RDC-V171051)

(13) La razón de esta elección hay que buscarla en el conocimiento cierto de que, a partir de la vigesimosexta edición, MARCA Plus no incorpora formatos diferentes de los juegos ofrecidos, lo que permite asegurar que los resultados extraídos de la muestra son cien por cien extrapolables al total de la publicación. (RDC-V181028)

El tercer movimiento observado en $79.6 \%$ de los artículos fue la descripción del análisis de los datos. En este, los autores describen y justifican los análisis seleccionados (68.9\%) tanto en estudios cuantitativos (ejemplo 14) como cualitativos (ejemplo 15).

(14) Se aplicaron herramientas de análisis multivariante correspondientes al análisis factorial para reducir las variables a componentes que expliquen la actividad de información sostenibilidad, pruebas ANOVA, así como de regresión lineal para determinar la significatividad de la influencia de los factores en el nivel de información. (RDC-V171046)

(15) Se analizan estas noticias y comentarios mediante triangulación de métodos y fuentes, usando técnicas cuantitativas y cualitativas: cómputo de shares y engagements en las principales redes, desde Buzzsumo; localización y significación de palabras más repetidas a través de Wordclouds, y de palabras clave; análisis e interpretación de los textos valorando percepciones sobre China positivas o negativas; seguimiento de creación de opinión en redes y elaboración de un grafo social, siguiendo la teoría de grafos, rama de las matemáticas discretas, utilizando la herramienta Gephi, que representa las relaciones entre usuarios. (RDC-V182011)

La estructura de esta sección difiere de lo observado en estudios previos en cuanto a los movimientos y pasos (Cotos et al. 22), lo cual habla de rasgos distintivos para el AI en comunicación. En el presente estudio, se observó que, si bien algunos movimientos no son 
muy frecuentes, aquellos orientados a la credibilidad y replicabilidad presentan porcentajes de aparición por encima de $65 \%$ al igual que lo observaron Kafes (142) y Cotos et al. (24).

\subsection{Resultados}

La sección de resultados mostró tres movimientos, de los cuales solo dos alcanzaron carácter obligatorio: reportar de los resultados por separado (94.4\%) (lo cual se hace describiendo e interpretando y, en algunos casos, discutiendo los mismos) y presentación de Tablas, y Figuras (77.8\%) (Tabla 4).

Tabla 4. Movimientos presentes en la sección de Resultados.

\begin{tabular}{lc}
\hline Movimiento & $\%$ \\
\hline Referencia a objetivo & 9.3 \\
Reportar resultados & 94.4 \\
Presenta Tablas y Gráficos & 77.8 \\
\hline
\end{tabular}

La presentación de los resultados en prosa, tablas e imágenes se complementaban entre sí y en ningún caso se observó repetición de información en más de uno de estos formatos. Un ejemplo de la descripción de un hallazgo se observa en el ejemplo (16), mientras que el ejemplo (17) refleja la forma en que el autor describe e interpreta el hallazgo.

(16)En nuestra encuesta, en la que cada respondente podía citar las tres más usuales, la red que aparece como más utilizada es WhatsApp (92,5\%), por lo que se acerca bastante al total. (RDC-V182011)

(17) Al ver el primer capítulo de la serie mediante una plataforma digital en el teléfono móvil se obtiene una curva con mucha dispersión y con crecimiento, aunque no muy pronunciado. Es decir, la atención no es tan constante como en otros dispositivos, lo que puede estar condicionado, precisamente, por la movilidad del propio soporte. (RDC-V182006)

En los artículos analizados, las tablas presentaban, mayormente, resultados de análisis descriptivos. Solo se usaron tablas en casos en que la cantidad de información lo ameritaba, por lo que se ve que estos autores manejan criterios claros en el uso de esta 
herramienta para la presentación de información. En cuanto al tipo de figuras que se usan, se observó un frecuente uso de fotografías, gráficos producto de herramientas de análisis cualitativo (gráficos de análisis de red, por ejemplo) e imágenes de escenas de obras cinematográficas. La presencia de gráficos estadísticos fue casi nula.

\subsection{Conclusiones}

Las conclusiones se presentaron como sección independiente en 66,7\% de los artículos. Los movimientos que componen esta sección y su frecuencia se detallan en la Tabla 5. Se observa que solo uno superó el 65\% señalado por Skelton (456), aunque otros movimientos se aproximaron a ese porcentaje.

Tabla 5. Movimientos presentes en la sección de conclusiones.

\begin{tabular}{lr}
\hline Movimiento & $\mathbf{\%}$ \\
\hline Información previa del estudio & 53.7 \\
Conclusión general & 63 \\
Conclusión por objetivo o hipótesis & $\mathbf{8 3 . 3}$ \\
Discutir con literatura previa & 25.9 \\
Implicaciones & 59.3 \\
Investigaciones futuras & 27.8 \\
\hline
\end{tabular}

La presentación de conclusiones en función de cada objetivo, hipótesis o categoría trabajada fue el movimiento más frecuente (83,3\%). En segundo lugar, aunque más bajo del porcentaje estipulado por Skelton (456) se observó el movimiento de presentar una conclusión general al inicio de la sección (63\%), implicaciones del estudio (59,3\%) y la apertura de la sección retomando el contexto de la investigación (53.7\%). Finalmente, las propuestas para futuras investigaciones estuvieron presente en muy pocos artículos. En aquellos que apareció este movimiento se hacía de forma explícita como se muestra en el ejemplo (18).

(18) Posteriores estudios se deben seguir haciendo sobre la cobertura periodística que se ha dado a la muerte de otros profesionales de la información. (RCDV171052) 


\subsection{Secciones complementarias}

La presencia de estas secciones fue muy escasa. Se observó que dos estudios presentaron secciones adicionales asociadas a las referencias, estas secciones fueron 'filmografía' y 'recursos web'. Estas secciones no habían sido observadas en otras disciplinas (Farnia y Rahimi 33; Morales et al. Estructura 387, por ejemplo). La sección de anexos estuvo presente en 9,3\%; estos eran instrumentos de recolección de datos. Los autores que incluyeron imágenes, fotos de escenas, copias de las noticias a analizar, entre otros, lo hicieron con los resultados para discutirlos, pero ninguno como anexo. Solo 9,1\% presentó sección de agradecimientos $\mathrm{y}$, en esta, se agradeció al ente financiador. Los expertos coincidieron que se incluyen secciones complementarias como agradecimientos y conflictos de intereses sólo bajo la exigencia de la revista.

\section{Discusión}

Este estudio constituye la primera aproximación de su tipo en el ámbito de la comunicación. Se ofrece lo que señala Skelton (456) como una descripción informada de probabilidades en la escritura dentro de esta comunidad discursiva, en lugar de una lista de indicaciones de cómo escribir. Como lo han planteado Cotos et al. (104), este tipo de estudios no pretende prescribir normas de escritura sino mostrar las prácticas asociadas a esta. En este sentido, lo recogido en este artículo resume una descripción de las prácticas de estos autores iberoamericanos en la escritura del AI en comunicación.

Se observan rasgos característicos en los $\mathrm{AI}$ en comunicaciones que lo diferencian del mismo género en otras comunidades. De esta forma, se confirma lo planteado por los autores que han argumentado que un mismo género presentará variaciones de una comunidad a otra (Bhatia Applied Genre 9, Critical, 51; Morales et al., 387; Saboori y Reza 493; Sánchez 21).

Las diferencias incluyeron la estructura de la introducción, la cual difiere de los hallazgos para el AI en odontología (Farnia y Rahimi 33) y lingüística aplicada (Santiago y Heredero 276), por ejemplo. Tomando como referencia el modelo CARS, en la introducción destacaron como obligatorios M1-P2, M2 y M3-P1A. Es decir, los autores establecen el territorio con base en generalizaciones sobre el tema; establecen el vacío 
haciendo de diferentes formas y presentan la propuesta para llenar el vacío por medio de la expresión del objetivo, hipótesis o supuestos.

La Metodología también presenta una estructura diferente a la hallada en estudios previos en AI en otras disciplinas (Zhang y Wannaruk 165 en educación; Kafes 142 en lingüística aplicada; Cotos et al. 104 en treinta disciplinas diferentes a la comunicación). Al contrastar con la estructura propuesta por Zhang y Wannaruk (165), los artículos analizados presentan los siguientes movimientos M1, M2 y M3, pero hay diferencias en los pasos para M2 y M3; además, el M1 inicia con la presentación del objetivo general; incluso muchos que ya lo habían enunciado en el M3 de la Introducción. En la sección de resultados hubo presencia de solos tres movimientos; dos de ellos obligatorios. De esta forma, la sección se conforma de los movimientos 'reportar resultados' y 'presentación de resultados en tablas y gráficos'.

La discusión no es una sección obligatoria (18.5\% del corpus). Este hallazgo difiere de estudios previos (Amnuay, The textual, 3). En el corpus analizado se notó que los autores de los AI de comunicación en español discutieron los resultados en la medida que iban presentando, por lo que pocos ven necesaria una sección destinada solo a la discusión. Los expertos señalaron que por la naturaleza de lo estudiado y de la metodología, esta práctica suele ser habitual por considerarse la más conveniente.

La conclusión es una sección obligatoria al igual que en otros estudios sobre AI (Ruiying y Allison 365; Skelton 455). Las conclusiones se presentan en función de los objetivos o hipótesis o categorías de análisis, independientemente de si se mencionan de forma explícita o no. Es decir, los autores van dando respuesta a cada interrogante y dando respuesta a cada planteamiento de investigación con que inician en la introducción o la metodología.

Por tratarse del primer estudio en su clase en el ámbito de las comunicaciones, se sugieren futuros estudios que permitan conocer otros rasgos conocer los mecanismos de posicionamiento y atenuación usados por los autores los cuales, debido a su importancia, también se han estudiado en otros ámbitos académicos (Chao 139; Meza, 3; Morales et al. Los mecanismos, 137). 


\section{Conclusiones}

El presente estudio representa una aproximación a las prácticas discursivas de la comunidad académica de la comunicación para la escritura del AI en español. A partir de los resultados, es posible fortalecer los programas de enseñanza de la lectura y escritura del AI con base en el conocimiento del género lo cual aumenta las posibilidades de éxito para investigadores nóveles.

La estructura predominante para el AI en comunicaciones en español publicados en revistas de impacto en Latinoamérica se resume en: Introducción-Metodología-ResultadosConclusiones (I-M-R-C). Aunque algunos movimientos y pasos se califican como opcionales, el porcentaje de aparición muestra la tendencia a obligatoriedad. La estructura retórica de los AI en comunicación en español, siguiendo los criterios establecidos en la literatura para su permanencia u obligatoriedad son:

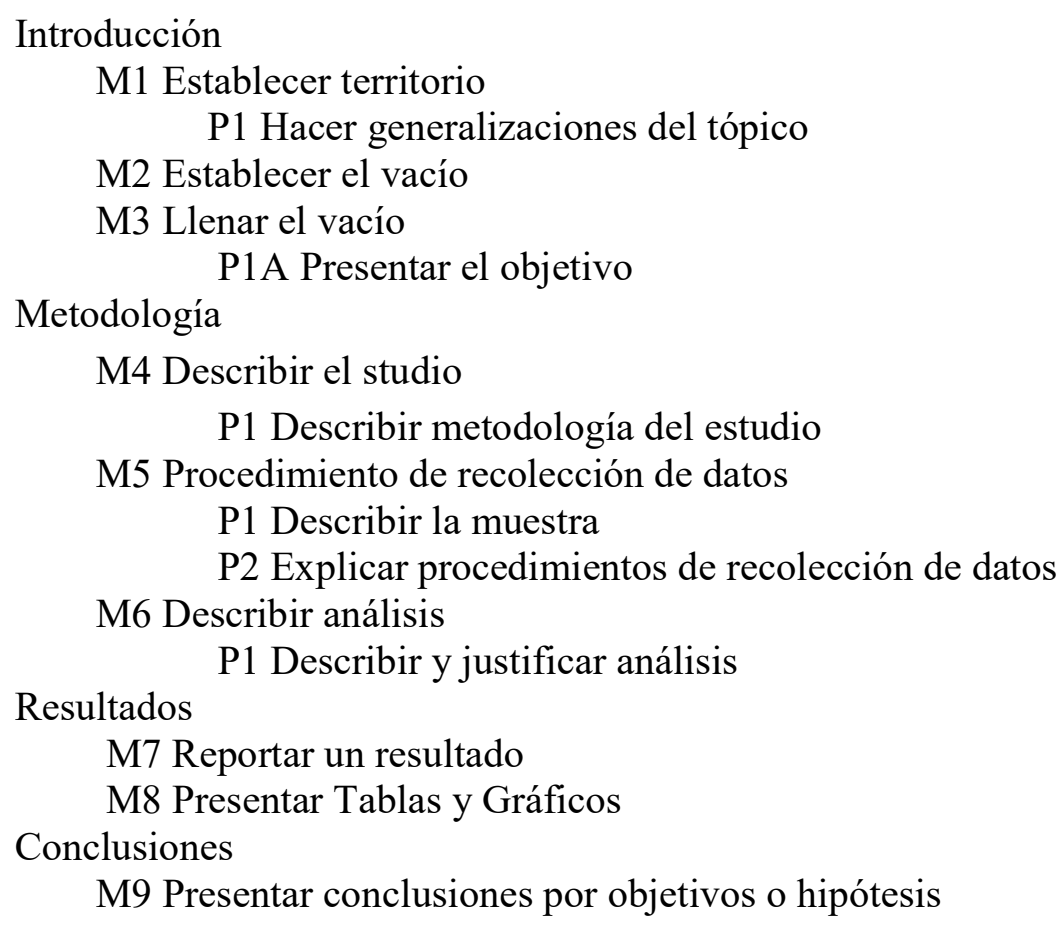

Los autores del área de comunicación investigan objetos y fenómenos diversos cuya naturaleza y complejidad difieren de los que se investigan en otras áreas, esto da espacio a la creatividad que se observa en la forma de concebir y presentar sus investigaciones. Por lo tanto, aunque existen directrices generales para la presentación de los AI, existe la libertad 
para denominar las secciones y estructurarlos como mejor se ajuste a su tema y elección metodológica. No obstante, independientemente de cómo les denominan, estas secciones ocupan el lugar y cumplen la función retórica correspondiente a estas secciones ampliamente conocidas como: introducción, metodología, resultados y conclusiones.

En futuras investigaciones sería oportuno realizar estudios contrastivos que contemplen el AI en español e inglés y diferentes especialidades del campo de la comunicación. De igual forma, se sugiere indagar sobre mecanismos como la atenuación y el posicionamiento.

Agradecimiento: Se hace público el agradecimiento a los informantes expertos, a los revisores anónimos de la versión previa de este manuscrito y al Centro de Investigación de la Creatividad (CIC) de la Universidad de Ciencias y Artes de América Latina (UCAL) por su apoyo para realizar este estudio.

Conflicto de intereses: La autora declara que no hubo conflictos de intereses relacionados con la investigación y el manuscrito.

\section{REFERENCIAS}

Abarghooeinezhad, Mahjoobeh y Shahla Simin. "Analyses of verb tense and voice of research article abstracts in engineering journals". International Letters of Social and Humanistic Sciences, núm. 47, 2015, pp. 139-152. DOI: 10.18052/www.scipress.com/ILSHS.47.139

Amnuai, Wiranda. "Analyses of Rhetorical Moves and Linguistic Realizations in Accounting Research Article Abstracts Published in International and Thai-Based Journals". SAGE Open, vol. 9, núm. 1, 2019, pp. 1-9. DOI: $10.1177 / 2158244018822384$

---. "The textual organization of the discussion sections of accounting research articles". Kasetsart Journal of Social Sciences, 2017, pp. 1-6. DOI: 10.1016/j.kjss.2017.10.007

Arias, Jesús, André Caldera, Óscar Alberto Morales, Bexi Perdomo, Daniel Cassany y Elix Izarra. "La estructura retórica de consentimientos informados usados en tesis y trabajos de grado de odontología en español". RLFE, núm. 26, 2020, pp. 149-166. https://ojsspdc.ulpgc.es/ojs/index.php/LFE/article/view/1091

Arroyave-Cabrera, Jesús, Rafael Repiso-Caballero y Rafael González-Pardo. "La investigación en Comunicación en Colombia vista desde Web of Science". Revista de Comunicación, vol. 19, núm. 2, 2020, pp. 29-45. https://doi.org/10.26441/RC19.2-2020-A2 
Artículo. Bexi Perdomo. “Análisis retórico-discurso del género artículo de investigación en español en el ámbito de la comunicación"

Berkenkotter, Carol. "Genre Systems at Work". Written Communication, vol. 18, núm. 3, 2001, pp. 326-349. https://dx.doi.org/10.1177/0741088301018003004

Bhatia, Vijay y Mohammad Salmani. "Genre analysis: The state of the art". International Journal of Language Studies, vol. 9, núm. 2, 2015, pp. 121-130. http://www.ijls.net/pages/volume/vol9no2.html

Bhatia, Vijay. “Applied genre analysis: a multi-perspective model”. Ibérica, núm. 4, 2002, pp. 3-19.

---. Critical Genre Analysis. Investigating interdiscursive performance in professional practice. Taylor \& Francis, 2017.

Campbell, Kim y Jefrey Naidoo. "Rhetorical Move Structure in High-Tech Marketing White Papers". Journal of Business and Technical Communication, vol. 31, núm. 1, 2016, pp. 94-118. DOI:10.1177/1050651916667532

Cárcamo, Benjamín. "Organización retórica y autorreferencia en el resumen de lingüística: Un estudio contrastivo inglés-español”. Lenguaje, vol. 47, núm. 2, 2019, pp. 334357. https://dx.doi.org/10.25100/lenguaje.v47i2.6748

Chao, Maikel. "Procedimientos de atenuación en los artículos de investigación científica: las directrices cognitivas en español". ELUA, Anexo 4, 2018, pp. 139-156. DOI: 10.14198/ELUA2018.ANEXO4.08

Compte-Pujol, Mark; Beatriz de Urquijo y Kathy Matilla. "La investigación en marcas de territorio y diplomacia pública en España. Un estudio bibliométrico de las revistas científicas españolas especializadas en Comunicación indexadas en Latindex (1980-2016)". Anales de Documentación, vol. 19, núm. 2, 2016. http://dx.doi.org/10.6018/analesdoc.19.2.255741.

Cotos, Elena, Sarah Huffman y Stephanie Link. "A move/step model for methods sections: Demonstrating Rigour and Credibility". ESP, núm. 46, 2017, pp. 90-106. DOI: 10.1016/j.esp.2017.01.001

Darabad, Ali. "Move Analysis of Research Article Abstracts: A Cross-Disciplinary Study". International Journal of Linguistics, vol. 8, núm. 2, 2016, pp. 125. DOI:10.5296/ijl.v8i2.9379

Estrada-Cuzcano, A. y J. Alhuay-Quispe. "Aproximación bibliométrica a la Revista de Comunicación (Perú), 2002-2019”. Revista de Comunicación, vol. 19, núm. 2, 2020, pp. 11-124. https://doi.org/10.26441/RC19.2-2020-A6

Farnia, Myriam y Shahrzad Rahimi. "Comparative Generic Analysis of Introductions of English and Persian Dentistry Research Articles". RELP, vol. 5, núm. 1, 2017, pp. 27-40. http://relp.khuisf.ac.ir/article_533644_042bfd33027afad954b4be3ee7f 5995c.pdf

Fox, Renate. "The Social Identity of Management Ergolect”. ESP, vol. 18, núm. 3, 1999, pp. 261-278. https://dx.doi.org/10.1016/s0889-4906(97)00057-4

Hanidar, Sharifah. "Rhetorical patterns, verb tense, and voice in cross disciplinary research article abstract". Humaniora, vol. 28, núm. 1, 2016, pp. 12-27. https://doi.org/10.22146/jh.11410

Hyland, Ken. "Genre: context, language and literacy". Annual Review of Applied Linguistics, núm. 22, 2002: pp. 113-135. https://doi.org/10.1017/ S0267190502000065

Kafes, Hüseyin. "Generic structure of the method sections of research articles and Ma theses by Turkish academic writers". International Journal of Language Academy, vol. 4, núm. 3, 2016, pp. 132-145. http://dx.doi.org/10.18033/ijla.429 
López, Daniel, Antonio Torres, Leticia Brito y María López. "Cómo redactar y organizar un artículo científico original". Revista de Especialidades Médico Quirúrgicas, núm. 19, 2014, pp. 236-243

Mateu-Arrom, Laura, Jhon Huguet, Carlos Errando, Alberto Breda y Joan Palou. "How to write an original article”. Actas Urológicas Españolas, vol. 42, núm. 9, 2018, pp. 545-550. https://doi.org/10.1016/j.acuro.2018.02.011

Meza, Paulina. "Estrategias de posicionamiento del Autor en Artículos de Investigación de Ciencias Sociales, Humanidades e Ingeniería: Novatos versus Expertos". Información tecnológica, vol. 29, núm. 2, 2018, pp. 318. https://dx.doi.org/10.4067/S0718-07642018000200003

Miller, Caroline. "Genre as Social Action (1984), Revisited 30 Years Later (2014)". Letras y Letras, vol. 31, núm. 3, 2015, pp. 56-72. DOI: 10.14393/LL63-v31n3a2015-5

Morales, Diego Adolfo, Gina Burdiles y Óscar Alberto Morales. "Los mecanismos de atenuación en cartas al editor. Una exploración en la comunidad médica". Nueva Revista del Pacífico, núm. 73, 2020, pp. 137-163. http://www.nuevarevistadelpacifico.cl/index.php/NRP/article/view/184

Morales, Oscar Alberto y Bexi Perdomo. "Escribir para publicar en la universidad: Una experiencia de alfabetización académica con estudiantes de odontología", EDUCERE, vol. 77, núm. 25, 2020, pp. 267-280.

Morales, Oscar Alberto, Bexi Perdomo, Daniel Cassany y Elix Izarra. "Estructura retórica de tesis y trabajos de grado en Odontología: un estudio en Hispanoamérica y España". Íkala, vol. 25, núm. 2, 2020, pp. 373-93. https://doi.org/10.17533/ udea.ikala.v25n02a06

Navarro, F. "Aportes para una didáctica de la escritura académica basada en géneros discursivos." D.E.L.T.A., vol. 35, núm. 2, 2019, pp. 1-32. http://dx.doi.org/10.1590/1678-460X2019350201

Ölçücü, Mahmut, Sezai Aydın y Yaşar Özgök. "How to Write and Publish a Scientific Article". Journal of Urology Surgery, vol. 5, núm. 2, 2018, pp. 63-67. DOI: $10.4274 /$ jus. 1623

Perdomo, Bexi y Óscar Alberto Morales. "Genre-Based Teaching in English for Specific Purposes: Systematic Review”. Revista Acción Pedagógica, vol. 27, núm. 1, 2018, pp. 66-75. http://www.saber.ula.ve/handle/123456789/46630

Perdomo, Bexi. "Enseñanza basada en el género y comprensión lectora en inglés con propósitos específicos: revisión sistemática". REDINE, vol. 10, núm. 1, 2018, pp. 29-41. https://revistas.uclave.org/index.php/redine/article/view/1214.

Perdomo, Bexi. y Katerine De Jong. "Inglés con propósitos académicos en el contexto de la Odontología Basada en la Evidencia”. RVIO, vol. 5, núm. 2, 2017, pp. 175-194. http://erevistas.saber.ula.ve/index.php/rvio/article/view/8225

Pérez, Adriana y Nayibe Rosado. "Pedagogía del género discursivo científico: una mirada desde una IES privada en Colombia”. Lengua y Habla, núm. 23, 2019, pp. 580600. http://erevistas.saber.ula.ve/index.php/lenguayhabla/article/view/15711

Piñeiro-Naval, Valeriano y Ricardo Morais. "Estudio de la producción académica sobre comunicación en España e Hispanoamérica". Comunicar, vol. XXVII, núm. 61, 2019, pp. 113-123. https://doi.org/10.3916/C61-2019-10

Ruiying, Yang y Desmond Allison. "Research articles in applied linguistics: Moving from results to conclusions". ESP, núm. 22, 2003, pp. 365-385. https://doi.org/10.1016/S0889-4906(02)00026-1 
Saboori, Fahimeh y Mohammad Reza. "A cross-disciplinary move analysis of research article abstracts". International Journal of Language Learning and Applied Linguistics World, vol. 4, núm. 4, 2013, pp. 483-496. https://profdoc.um.ac.ir/articles/a/1038627.pdf

Sal, Julio. "El comentario digital como género discursivo periodístico. Análisis de la gaceta de Tucumán”. Aposta. Revista de Ciencias Sociales, núm. 69, 2016, pp. 158-216. http://www.apostadigital.com/revistav3/hemeroteca/salpaz1.pdf

Sánchez, Alexánder. "Consideraciones sobre el artículo científico (AC): una aproximación desde el análisis de género y el posicionamiento". Lingüística y Literatura, núm. 73, 2018, pp. 17-36.

Santesteban-Echarri, Olga y Nuria Nuñez-Morales. "Cómo escribir un artículo científico por primera vez”. Psiquiatría Biológica, vol. 24, núm. 1, 2017, pp. 3-9. https://doi.org/10.1016/j.psiq.2017.01.004

Santiago, Gemma y David Heredero. "Las introducciones de artículos de investigación de lingüística y lingüística aplicada escritas por eslovenos". Colindancias: Revista de la Red de Hispanistas de Europa Central, núm. 9, 2018, pp. 271-291.

Skelton, John. "Analysis of structure of original research papers: an aid to writing original papers for publication". British Journal of General Practice, núm. 44, 1994, pp. 455-459. https://www.ncbi.nlm.nih.gov/pmc/articles/PMC1239019/

Soodmand, Hassan, Mehdi Doosti y Hossein Movassagh. "A Genre Analysis of the Introduction Section of Applied Linguistics and Chemistry Research Articles". IJAL, vol. 21, núm. 1, 2018, pp. 163-214.

Swales, John. The concept of Discourse community. Cambridge University Press, 1990.

Torres, Andrea. "¿Escritura disciplinar? Una experiencia a partir de la lectura crítica y la habilidad argumentativa en la universidad". Revista Mexicana de Investigación Educativa, vol. 23, núm. 76, 2018, pp. 95-124.

Wahyu, Leo. "Moves and grammatical constructions of research article abstracts". Journal of English for Academic and Specific Purposes, vol. 1, núm. 2, 2018, pp. 60-68.

Zare, Javad y Zahra Keivanloo-Shahrestanaki. "Genre awareness and academic lecture comprehension: The impact of teaching importance markers". Journal of English for Academic Purposes, núm. 27, 2017, pp. 31-41. doi:10.1016/j.jeap.2017.03.001

Zhang, Baoya y Anchalee Wannaruk. "Rhetorical Structure of Education Research Article Methods Sections”. PASAA, núm. 51, 2016, pp. 155-184. https://eric.ed.gov/?id=EJ1112248 